\title{
Effects of Ipragliflozin on Diabetic Nephropathy and Blood Pressure in Patients With Type 2 Diabetes: An Open-Label Study
}

\author{
Daisuke Ito ${ }^{\mathrm{a}, \mathrm{b}, \mathrm{f}}$, Emi Ikuma-Suwa ${ }^{\mathrm{a}}$, Kazuyuki Inoue ${ }^{\mathrm{a}, \mathrm{b}}$, Kimie Kaneko ${ }^{\mathrm{c}}$, Morifumi Yanagisawa ${ }^{\mathrm{b}, \mathrm{d}}$, \\ Kouichi Inukaie, Mitsuhiko Noda ${ }^{a}$, Akira Shimada ${ }^{\mathrm{a}}$
}

\begin{abstract}
Background: Sodium-glucose cotransporter 2 (SGLT2) inhibitors are novel agents used to treat type 2 diabetic patients. We investigated the efficacy of the SGLT2 inhibitor ipragliflozin on diabetic nephropathy in Japanese patients with type 2 diabetes.

Methods: A $50 \mathrm{mg}$ dose of ipragliflozin was administered for 24 weeks to 50 patients with type 2 diabetes who were concomitantly managed with diet and exercise therapy alone or antidiabetic medications other than SGLT2 inhibitors.

Results: At the end of the 24-week ipragliflozin treatment, significant decreases in mean glycated hemoglobin (HbAlc) $(1.0 \pm 1.2 \%)$ and body weight $(2.7 \pm 2.5 \mathrm{~kg})$ were observed; in addition, median urinary albumin-to-creatinine ratio (UACR) significantly decreased from $15.5(8.0$ - 85.7) to $12.9(7.4$ - 36.3) $\mathrm{mg} / \mathrm{gCr}$. Sub-analysis by renal function at baseline revealed that median UACR in patients with estimated glomerular filtration rate (eGFR) $\geq 90 \mathrm{~mL} / \mathrm{min} / 1.73$ $\mathrm{m}^{2}$ decreased significantly from $12.3(7.5$ - 89.6) to $10.6(5.8$ - 27.3) $\mathrm{mg} / \mathrm{gCr}$. Furthermore, mean eGFR decreased significantly from 102.4 \pm 8.6 to $93.6 \pm 10.5 \mathrm{~mL} / \mathrm{min} / 1.73 \mathrm{~m}^{2}$ in these patients. In contrast, UACR and eGFR did not change significantly in patients with eGFR $<90$. In addition, analysis of the relationship between the amount of change in UACR and blood pressure at 24 weeks revealed a significant positive correlation between UACR and SBP values, independently of the presence of diabetic nephropathy.
\end{abstract}

Conclusions: Our results indicate that ipragliflozin may facilitate $\mathrm{HbA1c}$ control and body weight reduction. Furthermore, our results

Manuscript accepted for publication December 20, 2016

aDepartment of Endocrinology and Diabetes, Saitama Medical University, Saitama, Japan

${ }^{b}$ Department of Internal Medicine, Ogawa Red Cross Hospital, Saitama, Japan 'Department of Nursing, Ogawa Red Cross Hospital, Saitama, Japan

dSatsuki Medical Clinic, Saitama, Japan

eDepartment of Diabetes and Endocrinology, Higashiyamato Hospital, Tokyo, Japan

${ }^{f}$ Corresponding Author: Daisuke Ito, Department of Endocrinology and Diabetes, Saitama Medical University, 38, Morohongo, Moroyama, Iruma-gun, Saitama 350-0495, Japan. Email: dito@saitama-med.ac.jp

doi: https://doi.org/10.14740/jocmr2875w also raise the possibility that ipragliflozin significantly reduces urinary albumin levels and improves glomerular hyperfiltration in a subset of patients with type 2 diabetes.

Keywords: Ipragliflozin; Sodium-glucose cotransporter 2 inhibitor; Diabetic nephropathy; Blood pressure; Type 2 diabetes

\section{Introduction}

In recent years, the number of patients with type 2 diabetes has increased in parallel with the increasing rate of obesity in Japan. Thus, the clinical management of diabetic patients is currently focused on the importance of therapeutic approaches to suppressing obesity while achieving blood glucose control. Among such treatment modalities, sodium-glucose cotransporter 2 (SGLT2) inhibitors were shown to have good blood glucoselowering effects in addition to their ability to control obesity [1-4]. Thus, SGLT2 inhibitors are widely expected to play a critical role in the comprehensive management of diabetes.

Diabetic nephropathy is a particularly serious microvascular complication of diabetes. Several clinical trials have demonstrated the efficacy of SGLT2 inhibitors in patients with diabetic nephropathy in recent years [5-7], with studies in diabetic animal models also indicating the renoprotective effects of SGLT2 inhibitors [8-14]. Additionally, EMPA-REG OUTCOME, a long-term prospective clinical trial, revealed that empagliflozin, an SGLT2 inhibitor, exhibited protective effects against macrovascular and renal events $[7,15]$. Although SGLT2 inhibitors were highly anticipated to exert renoprotective effects, the question of whether this benefit extends to all SGLT2 inhibitors remains unclear. Although several reports have demonstrated improvements in blood glucose levels and weight reduction in patients treated with ipragliflozin, an SGLT2 inhibitor first approved for use in Japan [16-20], there have as yet been no studies examining its beneficial effects on diabetic nephropathy.

In the present study, we examined the effects of long-term treatment with ipragliflozin in patients with type 2 diabetes by evaluating several parameters, including blood glucose and body weight, with a particular focus on its impact on diabetic nephropathy and blood pressure. The findings of this study suggest that ipragliflozin exerts beneficial effects on diabetic 
nephropathy in a subset of patients with type 2 diabetes, thereby establishing a clear rationale for the use of SGLT2 inhibitors to prevent diabetic complications.

\section{Materials and Methods}

\section{Study design}

This was a multicenter, open-label, 24-week study to evaluate the efficacy and safety of a $50 \mathrm{mg}$ dose of ipragliflozin in patients with type 2 diabetes. Glycemic and metabolic parameters, diabetic nephropathy and blood pressure were examined. This study included participants from three hospitals in Japan and was conducted between May 2014 and April 2016, based on the ethical principles outlined in the Declaration of Helsinki. Informed consent was obtained from all patients. This trial was registered with UMIN-CTR (UMIN000022615).

\section{Patients}

Patients were between 20 and 75 years of age, with glycated hemoglobin (HbA1c) (NGSP) values of 6.5-11.0\% and a body mass index (BMI) of $22-45 \mathrm{~kg} / \mathrm{m}^{2}$. Type 2 diabetic patients who were treated with diet and exercise therapy alone or antidiabetic drugs other than SGLT2 inhibitors, with or without insulin, were eligible to participate in this study. The main exclusion criteria included history of serious diabetic complications, findings suggestive of insulin dependency, heart failure (New York Heart Association Class III or IV), history of myocardial or cerebral infarction, estimated glomerular filtration rate $(\mathrm{eGFR})<45 \mathrm{~mL} / \mathrm{min} / 1.73 \mathrm{~m}^{2}$, serum creatinine $\geq 1.5 \mathrm{mg} /$ $\mathrm{dL}$, and hepatic dysfunction.

\section{Treatment}

Eligible patients were treated with $50 \mathrm{mg}$ of ipragliflozin once daily for 24 weeks, with no dose adjustments. Patients who were already being treated for diabetes at the time of enrollment continued their prior treatments during the study period, and ipragliflozin was added to the current regimen. During the study, no additions or adjustments were made to current antidiabetic drugs, antihypertensive medications, including angiotensin receptor blocker (ARB) and angiotensin-converting enzyme (ACE) inhibitor, or anti-lipidemic agents. However, it was permissible to reduce the doses of sulfonylurea and insulin, and even to discontinue these medications, if necessary to avoid any risk of hypoglycemia. During the observation period, all patients received $50 \mathrm{mg}$ of ipragliflozin once daily, without dosage adjustments.

\section{Assessments}

At the beginning of the study, all patients underwent physical examination, vital sign examination, clinical laboratory tests, and measurement of body weight, HbA1c, fasting plasma glucose, serum insulin, serum C-peptide, and serum lipid levels as well as urinary albumin-to-creatinine ratio (UACR), serum total adiponectin, and eGFR. This study also included the homeostasis model assessment (HOMA) of insulin resistance (HOMA-IR) and $\beta$-cell function (HOMA- $\beta$ ). Abdominal computed tomography (CT) without contrast was performed to measure visceral and subcutaneous fat areas. Clinical laboratory tests were performed at all outpatient visits during the study period, and non-contrast CT scans to measure visceral and subcutaneous fat areas were obtained at the end of the 24week treatment period. All laboratory tests were performed at SRL Laboratories (SRL Inc., Tokyo, Japan).

\section{Safety}

Adverse events and safety assessments, including symptoms or signs of hypoglycemia, vital signs, 12-lead electrocardiography and clinical laboratory tests were regularly recorded throughout the study.

\section{Statistical analysis}

Data were presented as mean \pm standard deviation (SD) or medians with the interquartile range (IQR). Statistical analysis was performed by means of appropriate parametric and nonparametric methods. Changes in continuous measures between baseline and post-treatment periods were tested by means of a paired $t$-test. Non-parametric methods were used for nonnormally distributed values. Changes from baseline in UACR at week 24 were analyzed using the Wilcoxon signed-rank test. The relationship between change in UACR and blood pressure was assessed using Pearson's correlation coefficients. All analyses were performed with SPSS Statistics, version 22.0 (IBM Corp., Armonk, USA). A P-value $<0.05$ was considered statistically significant.

\section{Results}

\section{All patients}

The baseline characteristics of all patients at the beginning of the study are shown in the left column of Table 1. In total, 50 patients who were eligible to participate in the study were treated with $50 \mathrm{mg}$ of ipragliflozin and followed up for 24 weeks. Table 2 shows the changes in parameters between baseline and 24 weeks of ipragliflozin treatment. Significant reductions in $\mathrm{HbA1c}$, fasting plasma glucose, and body weight were observed $(8.7 \pm 1.5 \%$ vs. $7.7 \pm 1.2 \%, 165.5 \pm 40.5$ vs. $137.6 \pm 25.6 \mathrm{mg} / \mathrm{dL}$, and $78.4 \pm 19.7$ vs. $75.8 \pm 20.1 \mathrm{~kg}$, respectively; $\mathrm{P}<0.001)$. Additionally, there were significant decreases in visceral and subcutaneous fat areas at the end of 24 weeks compared to baseline $\left(152.1 \pm 72.2\right.$ vs. $124.5 \pm 54.0 \mathrm{~cm}^{2}(18.1 \%$ reduction $)$ and 279.3 \pm 154.2 vs. $252.0 \pm 144.2 \mathrm{~cm}^{2}(9.8 \%$ reduction $)$, respectively; $\mathrm{P}<0.001)$. Median UACR significantly decreased from 15.5 
Table 1. Baseline Characteristics

\begin{tabular}{|c|c|c|}
\hline & All patients & Diabetic nephropathy \\
\hline Number of patients & 50 & 16 \\
\hline Male/female (n) & $16 / 34$ & $6 / 10$ \\
\hline Age (years) & $53.2 \pm 12.5$ & $53.8 \pm 13.3$ \\
\hline Duration of diabetes (years) & $9.1 \pm 6.0$ & $9.9 \pm 7.2$ \\
\hline Body weight (kg) & $78.4 \pm 19.7$ & $82.9 \pm 15.7$ \\
\hline Body mass index $\left(\mathrm{kg} / \mathrm{m}^{2}\right)$ & $30.6 \pm 6.2$ & $32.1 \pm 4.5$ \\
\hline HbA1c (NGSP) (\%) & $8.7 \pm 1.5$ & $9.0 \pm 1.8$ \\
\hline Fasting plasma glucose (mg/dL) & $165.5 \pm 40.5$ & $162.5 \pm 32.1$ \\
\hline Fasting C-peptide (ng/mL) & $3.4 \pm 1.8$ & $3.5 \pm 1.03$ \\
\hline eGFR (mL/min/1.73 m²) & $82.1 \pm 19.8$ & $77.9 \pm 20.2$ \\
\hline UACR (mg/gCr) & $15.5(8.0-85.7)$ & $119.2(98.9-201.8)$ \\
\hline Systolic blood pressure (mm Hg) & $131.8 \pm 13.1$ & $135.5 \pm 17.2$ \\
\hline Diastolic blood pressure (mm Hg) & $80.7 \pm 11.0$ & $83.9 \pm 12.4$ \\
\hline \multicolumn{3}{|l|}{ Medications } \\
\hline Metformin $(\%)$ & $31(62.0 \%)$ & $10(62.5 \%)$ \\
\hline DPP-4 inhibitor (\%) & $31(62.0 \%)$ & $8(50.0 \%)$ \\
\hline Sulfonylurea (\%) & $10(20.0 \%)$ & $2(12.5 \%)$ \\
\hline Insulin $(\%)$ & $12(24.0 \%)$ & $5(31.3 \%)$ \\
\hline ARB or ACE inhibitor (\%) & $32(64.0 \%)$ & $13(81.3 \%)$ \\
\hline Statin $(\%)$ & $32(64.0 \%)$ & $10(62.5 \%)$ \\
\hline
\end{tabular}

Data are $\mathrm{n}(\%)$, means \pm standard deviation (SD), or medians with the interquartile range (IQR). HbA1c: glycated hemoglobin; eGFR: estimated glomerular filtration rate; UACR: urinary albumin-to-creatinine ratio; DPP-4: dipeptidyl peptidase-4; ARB: angiotensin receptor blocker; ACE: angiotensin-converting enzyme.

$(8.0$ - 85.7) to $12.9(7.4-36.3) \mathrm{mg} / \mathrm{gCr}(\mathrm{P}=0.011)$, and mean eGFR decreased significantly from $82.1 \pm 19.8$ to $78.5 \pm 17.4$ $\mathrm{mL} / \mathrm{min} / 1.73 \mathrm{~m}^{2}(\mathrm{P}=0.007)$. No significant changes in either systolic blood pressure (SBP) or diastolic blood pressure (DBP) were found between baseline and after 24 weeks of treatment $(131.8 \pm 13.1$ vs. $130.1 \pm 13.0 \mathrm{~mm} \mathrm{Hg}$ and $80.7 \pm 11.0$ vs. 80.8 $\pm 10.5 \mathrm{~mm} \mathrm{Hg}$, respectively (Table 2 , left column).

\section{Subgroup analysis based on renal function}

Figure 1 shows the subgroup analysis results for patients categorized according to their renal function at baseline into three groups: high $(\mathrm{H}, \mathrm{eGFR} \geq 90, \mathrm{n}=20)$, medium $(\mathrm{M}, \mathrm{eGFR} \geq$ 60 to $<90, \mathrm{n}=22$ ), and low (L, eGFR $<60, \mathrm{n}=8$ ). After 24 weeks of treatment with ipragliflozin, HbA1c values in the $\mathrm{H}$ and $\mathrm{M}$ groups had significantly decreased from $9.4 \pm 1.6 \%$ to $7.9 \pm 1.3 \%(\mathrm{P}<0.001)$ and from $8.1 \pm 1.2 \%$ to $7.4 \pm 0.8 \%(\mathrm{P}=$ $0.009)$, respectively. However, no changes were noted in the L group (Fig. 1a). The body weights of patients in all groups were significantly decreased after 24 weeks (mean losses of $2.6,2.7$, and $2.3 \mathrm{~kg}$ in the $\mathrm{H}, \mathrm{M}$, and L groups, respectively), regardless of baseline renal function (Fig. 1b). The median UACR was significantly decreased from $12.3(7.5-89.6)$ to $10.6(5.8-27.3) \mathrm{mg} / \mathrm{gCr}$ only in the $\mathrm{H}$ group $(\mathrm{P}=0.003)$; no significant difference in the median UACR was noted in either the $\mathrm{M}$ or the L group (Fig. 1d). Similarly, although the mean eGFR was significantly decreased in the $\mathrm{H}$ group, from 102.4 \pm 8.6 to $93.6 \pm 10.5 \mathrm{~mL} / \mathrm{min} / 1.73 \mathrm{~m}^{2}(\mathrm{P}<0.001)$, the $\mathrm{M}$ and $\mathrm{L}$ groups showed no significant changes in mean eGFR (Fig. 1c). Finally, we observed non-significant differences in SBP in the $\mathrm{H}$ and $\mathrm{M}$, but not the L, groups (Fig. 2).

\section{Subgroup analysis based on diabetic nephropathy}

Table 1 (right column) shows the baseline characteristics of 16 patients with diabetic nephropathy enrolled in this study, as determined by the presence of microalbuminuria (UACR $\geq 30$ $\mathrm{mg} / \mathrm{gCr}$ ). Table 2 (right column) shows the analysis of these patients after 24 weeks. After 24 weeks of ipragliflozin treatment, both mean $\mathrm{HbA} 1 \mathrm{c}$ and body weight were significantly decreased $(9.0 \pm 1.8 \%$ vs. $8.0 \pm 1.2 \%$ and $82.9 \pm 15.7$ vs. $80.2 \pm$ $15.9 \mathrm{~kg}$, respectively) in all patients with diabetic nephropathy after 24 weeks. Additionally, median UACR significantly decreased from 119.2 (98.9 - 201.8) to $52.9(24.8$ - 242.1) $\mathrm{mg} /$ $\mathrm{gCr}(\mathrm{P}=0.049)$. However, the decreases observed in eGFR $\left(77.9 \pm 20.2\right.$ vs. $\left.73.3 \pm 16.9 \mathrm{~mL} / \mathrm{min} / 1.73 \mathrm{~m}^{2}\right)$ in this subgroup did not reach statistical significance.

Next, patients with nephropathy were categorized into two groups: those with high eGFR $(\mathrm{NH}, \mathrm{eGFR} \geq 80, \mathrm{n}=7)$ and those with low eGFR (NL, eGFR $<80, \mathrm{n}=9$, Fig. 3). After 
Table 2. Changes in the Characteristics of Patients Between Baseline and Week 24

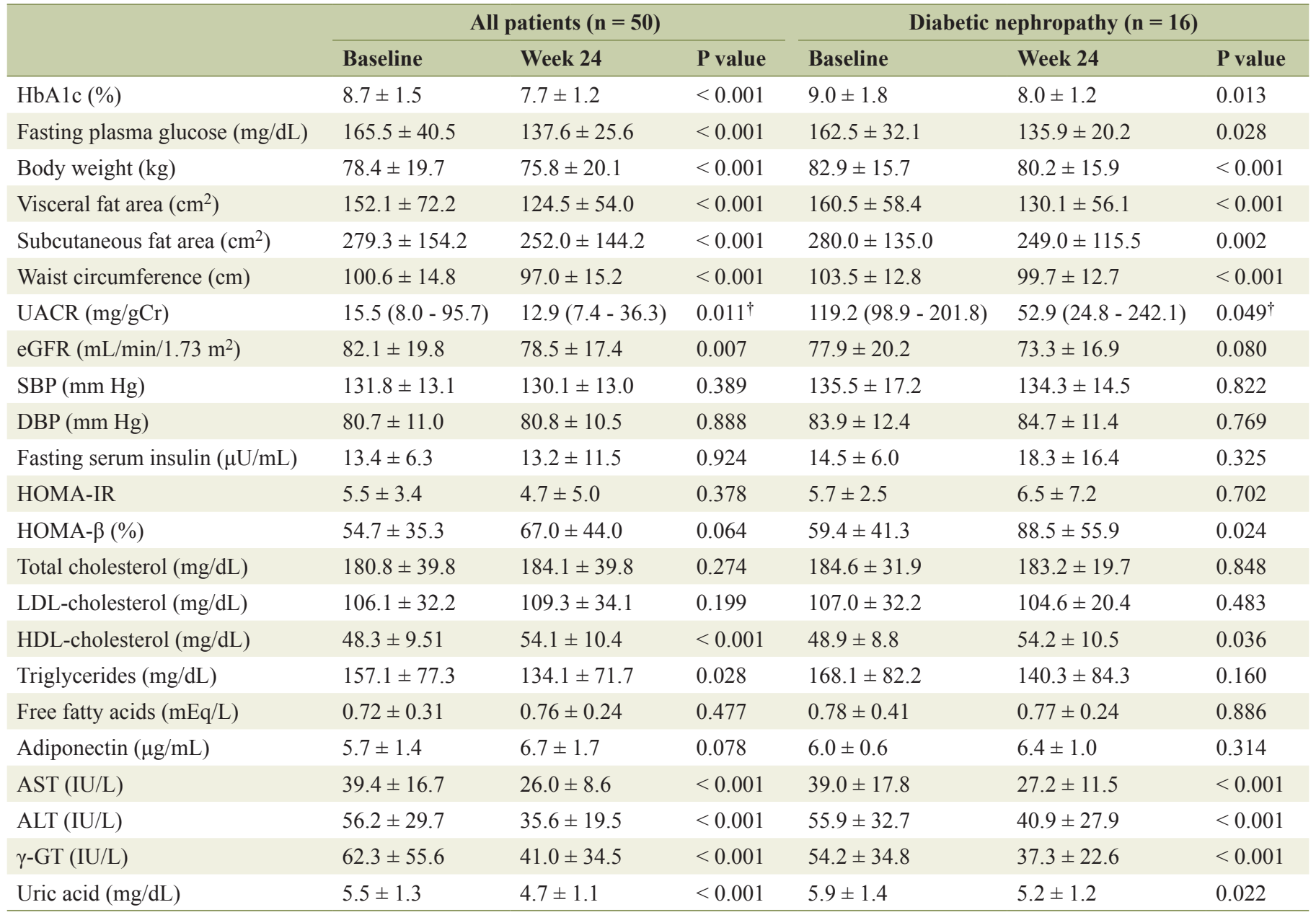

Data are means \pm standard deviation (SD) or medians with the interquartile range (IQR). P value versus baseline by the paired $t$-test. ${ }^{t} \mathrm{P}$ value versus baseline by the Wilcoxon signed-rank test. HbA1c: glycated hemoglobin; eGFR: estimated glomerular filtration rate; UACR: urinary albumin-to-creatinine ratio; SBP: systolic blood pressure; DBP: diastolic blood pressure; HOMA-IR: homeostasis model assessment of insulin resistance; HOMA- $\beta$ : homeostasis model assessment- $\beta$; LDL-cholesterol: low-density lipoprotein cholesterol; HDL-cholesterol: high-density lipoprotein cholesterol; AST: aspartate aminotransferase; ALT: alanine aminotransferase; $\gamma$-GT: $\gamma$-glutamyltransferase.

24 weeks, HbA1c exhibited a decreasing tendency and body weight was significantly decreased in both groups. Importantly, we observed significant decreases in median UACR (106.7 (92.3 - 274.3) vs. $47.0(35.7-102.3) \mathrm{mg} / \mathrm{gCr}, \mathrm{P}=0.018)$ and mean eGFR $\left(97.8 \pm 9.5\right.$ vs. $87.3 \pm 9.1 \mathrm{~mL} / \mathrm{min} / 1.73 \mathrm{~m}^{2}, \mathrm{P}=$ 0.050 ) only in the NH group.

Interestingly, a significant decrease in mean UACR, from $527.0 \pm 254.8$ to $310.2 \pm 220.0 \mathrm{mg} / \mathrm{gCr}(\mathrm{P}=0.031)$, was noted in four patients with pre-treatment UACR $\geq 300 \mathrm{mg} / \mathrm{gCr}$ (i.e., those with overt albuminuria). Incidentally, median UACR exhibited a decreasing tendency from 531.6 (380.7 - 677.8) to 314.6 (139.7 - 485.1) mg/gCr $(\mathrm{P}=0.068)$. These four patients showed no changes in eGFR.

\section{Patients with uncontrolled blood pressure}

There were no significant changes in blood pressure levels in any of the patients enrolled in this study. However, subgroup analysis of 23 patients with poorly controlled blood pressure, defined by an SBP $\geq 140 \mathrm{~mm} \mathrm{Hg}$ and/or a DBP $\geq 90 \mathrm{~mm} \mathrm{Hg}$, showed significant decreases in SBP (141.3 \pm 9.8 vs. $134.7 \pm$ $12.7 \mathrm{~mm} \mathrm{Hg}, \mathrm{P}=0.035)$ and $\mathrm{DBP}(88.1 \pm 9.2$ vs. $85.1 \pm 10.7$ $\mathrm{mm} \mathrm{Hg}, \mathrm{P}=0.045$ ) following 24 weeks of ipragliflozin treatment.

\section{The relationship between diabetic nephropathy and blood pressure}

Analysis of the relationship between the amount of change in UACR and blood pressure at 24 weeks after the start of treatment revealed a significant positive correlation between UACR and SBP values, independently of the presence of diabetic nephropathy (Fig. 4). This suggested that the SBP-lowering effect of ipragliflozin was a factor in its beneficial effects 

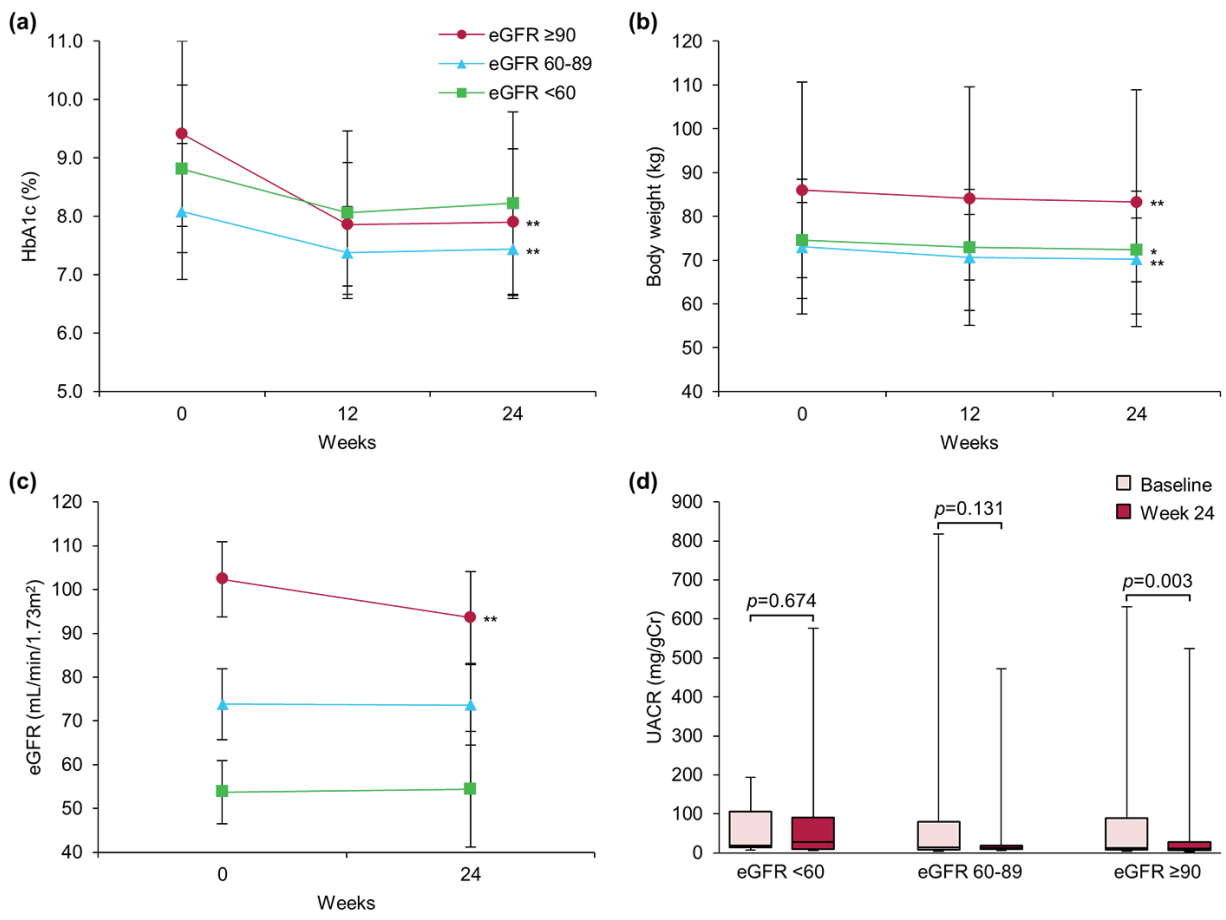

Figure 1. Effect of ipragliflozin on efficacy parameters at week 24 in all patients $(n=50)$, classified according to estimated glomerular filtration rate (eGFR) at baseline into three groups: eGFR $\geq 90$ (circles; $n=20$ ), eGFR $\geq 60$ to $<90$ (triangles; $n=22$ ), and eGFR < 60 (squares; $n=8$ ). (a) Change from baseline in glycated hemoglobin (HbA1c) to week 24 after starting ipragliflozin administration. (b) Change from baseline in body weight. (c) Change from baseline in eGFR. (d) Change from baseline in urinary albumin-to-creatinine ratio (UACR) at week 24. Data are means \pm standard deviation (SD) or medians with the interquartile range (IQR). ${ }^{*} \mathrm{P}<0.05,{ }^{* *} \mathrm{P}<0.01$ versus baseline by the paired $t$-test. For the UACR, the Wilcoxon signed-rank test was used to evaluate changes between the baseline and week 24. Error bars show SDs or IQRs.

on diabetic nephropathy.

\section{Additional efficacy evaluations}

Table 2 shows changes observed in other efficacy parameters after 24 weeks of ipragliflozin treatment. Significant improvements in serum lipid profiles, hepatic function, and uric acid values occurred in all patients, irrespective of the presence of diabetic nephropathy. Interestingly, although the beneficial effects of ipragliflozin on $\mathrm{HbA1c}$ were not statistically significant, even as eGFR decreased, improvements in the other efficacy parameters were maintained despite these decreases in eGFR.

\section{Safety}

During the present study, urinary tract infection, vaginal candidiasis, and transient loose stools were experienced by one, two, and two female subjects, respectively. However, these adverse effects resolved with administration of appropriate antibiotics or antifungals, and all five patients were able to continue the study. There were no other adverse events such as hypoglycemia, dehydration, ketoacidosis, cardiac failure, or severe infection necessitating treatment interruption or termi- nation.

\section{Discussion}

Glomerular hyperfiltration plays a crucial role in the onset and progression of diabetic nephropathy. In a hyperglycemic state, larger than normal amounts of glucose filtered by glomeruli flow into the proximal convoluted tubule (PCT). As SGLT2 expression and glucose uptake in the PCT are significantly enhanced in diabetic patients [21], $\mathrm{NaCl}$ reabsorption into the PCT is concurrently increased. As a result, $\mathrm{NaCl}$ passing through the ascending limb of the PCT and reaching the macula densa is decreased, which attenuates the release of adenosine that acts as a vasoconstrictor and subsequently enhances resistance in the afferent arteriole, while GFR in individual nephrons increases. Thus, the breakdown of tubuloglomerular feedback (TGF) in response to glomerular hyperfiltration occurs in diabetic patients. In contrast, inhibition of SGLT2 increases the delivery of $\mathrm{NaCl}$ to the macula densa and activates TGF, with contraction of afferent arterioles and improved hyperfiltration. In practice, the assessment of GFR values before and after glucose clamp performed after 8 weeks of empagliflozin treatment in patients with type 1 diabetes showed that GFR normalized after administration of this drug only in patients with hyperfiltration [6].

Unlike the previous empagliflozin study, our present study 

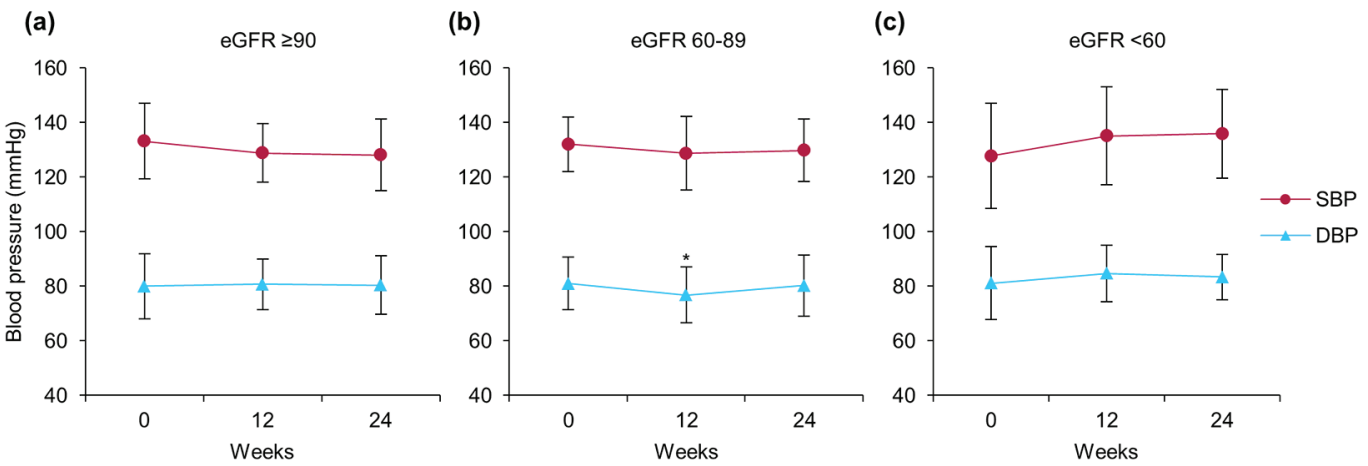

Figure 2. Changes in systolic blood pressure (circles) and diastolic blood pressure (triangles) from baseline to week 24 after starting ipragliflozin administration, classified according to estimated glomerular filtration rate (eGFR): (a) eGFR $\geq 90(n=20)$, (b) eGFR $\geq 60$ to $<90(n=22)$, and (c) eGFR $<60(n=8)$ at baseline. Data are means \pm standard deviation (SD). ${ }^{*} P<0.05$ versus baseline by the paired $t$-test. Error bars show SDs.

was conducted in patients with type 2 diabetes and also with a longer administration period. However, the findings presented herein were similar to those obtained in the prior empagliflozin study as significant decreases in eGFR and urinary albumin were observed only in patients with an eGFR of $\geq 90 \mathrm{~mL} /$ $\mathrm{min} / 1.73 \mathrm{~m}^{2}$ at baseline (mean eGFR $102.4 \mathrm{~mL} / \mathrm{min} / 1.73 \mathrm{~m}^{2}$ ). In addition, proteinuria was significantly reduced in only four patients in the present study, raising the possibility that overt albuminuria could be improved with ipragliflozin. However, at this time, the mechanism underlying these changes cannot be at- tributed to only the aforementioned amelioration of TGF failure.

To date, the effects of SGLT2 inhibitors on diabetic nephropathy have been demonstrated in animal studies using various inhibitors and models of disease [8-14]. Among those investigated, empagliflozin was found to improve glomerular hyperfiltration independently of blood glucose in a type 1 diabetes model animal, Akita mice, and was reported to suppress the renal expressions of inflammatory genes, urinary albumin excretion, and kidney weight [11]. This suggests that SGLT2 inhibitor-mediated effects on diabetic nephropathy occurred
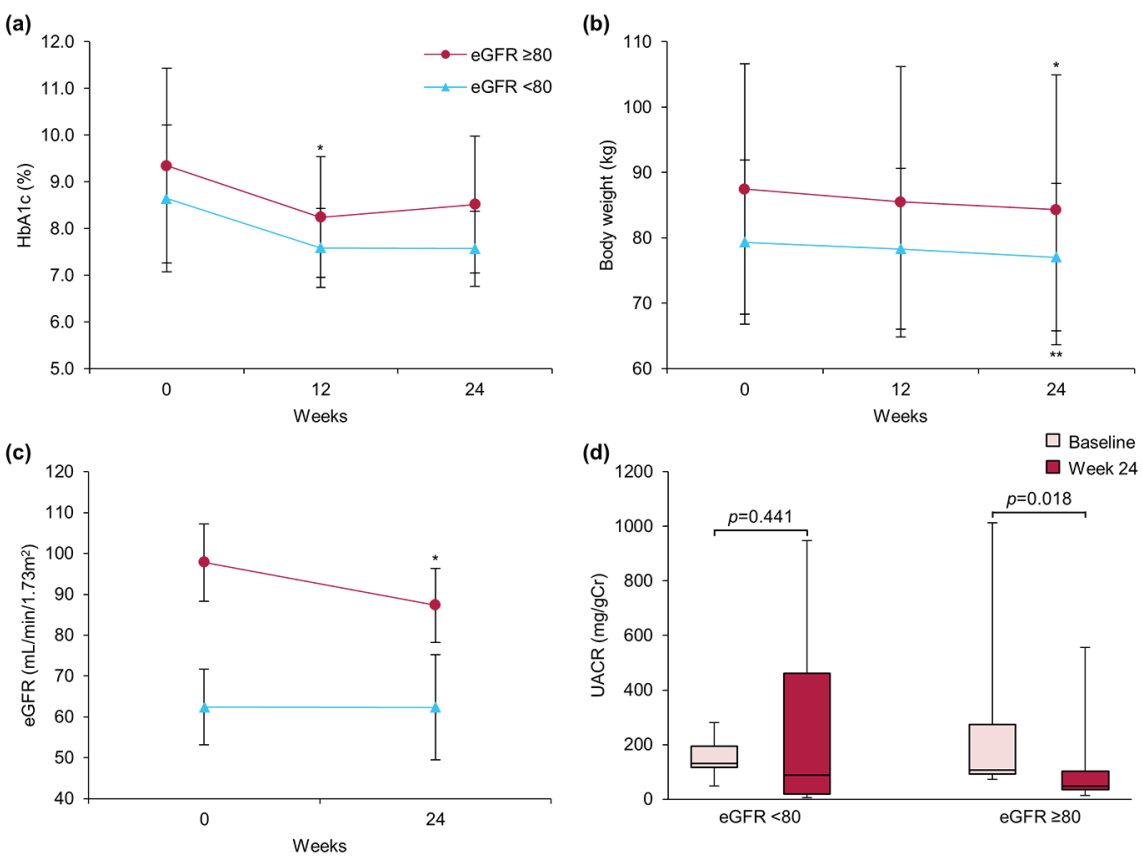

Figure 3. Effect of ipragliflozin on efficacy parameters at week 24 in patients with diabetic nephropathy $(n=16)$, classified according to estimated glomerular filtration rate (eGFR) at baseline into two groups: eGFR $\geq 80$ (circles; $n=7$ ) and eGFR $<80$ (triangles; $n=9$ ). (a) Change from baseline in glycated hemoglobin (HbA1c) to week 24 after starting ipragliflozin administration. (b) Change from baseline in body weight. (c) Change from baseline in eGFR. (d) Change from baseline in urinary albumin-tocreatinine ratio (UACR) at week 24 . Data are means \pm standard deviation (SD) or medians with the interquartile range (IQR). *P $<0.05$ versus baseline by the paired $t$-test. For the UACR, the Wilcoxon signed-rank test was used to evaluate changes between the baseline and week 24. Error bars show SDs or IQRs. 

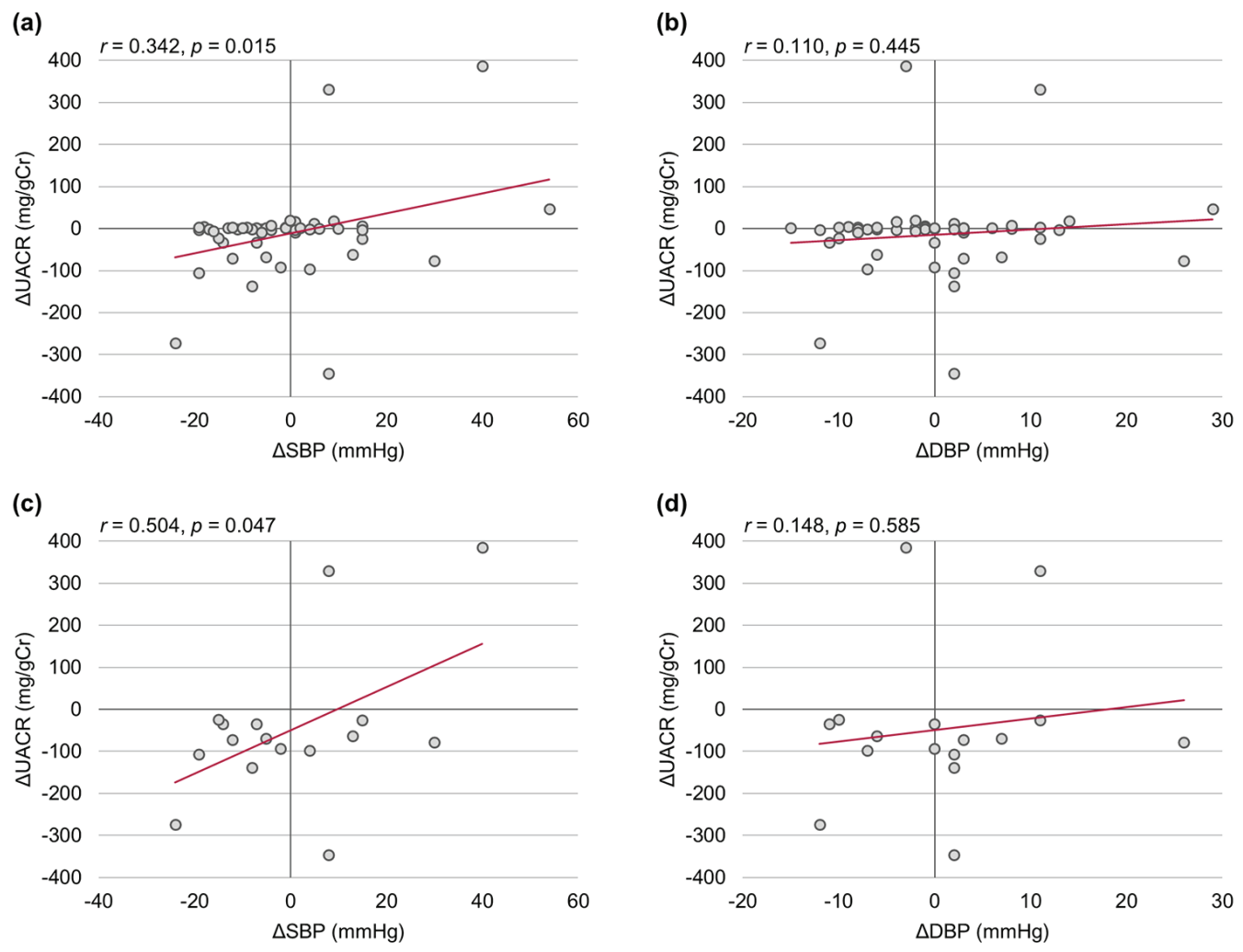

Figure 4. Correlations among urinary albumin-to-creatinine ratio (UACR), systolic blood pressure (SBP), and diastolic blood pressure (DBP). $(a, b)$ All patients $(n=50)$. (c, d) Patients with diabetic nephropathy $(n=16)$. The relationship between change in UACR and blood pressure was assessed using Pearson's correlation coefficients. $\Delta$ : the amount of change 24 weeks after baseline.

via blood glucose reduction and TGF activation, in addition to yet-unknown mechanisms in the kidneys. A recent report also showed that hyperglycemic conditions led to the contraction and hypertrophy of mesangial cells in rat [22]. This report demonstrated SGLT2 to be ectopically expressed in mesangial cells. Moreover, contraction of mesangial cells induced by intracellular $\mathrm{Ca}^{2+}$ influx regulated via both SGLT2 and the sodiumcalcium exchanger NCX, was shown to be inhibited by SGLT2 inhibitors, which suggested a direct protective role of SGLT2 inhibitors in the kidneys of patients with diabetic nephropathy.

SGLT2 inhibitors were also shown to exert antihypertensive effects. A phase III clinical trial of the SGLT2 inhibitor, dapagliflozin, in treatment-naive patients demonstrated slight increases in SBP and DBP in the placebo group and decreases in SBP and DBP (max. 4.6 and $2.0 \mathrm{~mm} \mathrm{Hg}$, respectively) in the treatment group receiving $1-5 \mathrm{mg}$ /day dapagliflozin [23]. A systematic review and meta-analysis investigating the effects of SGLT2 inhibitors also showed significant decreases in SBP and DBP (3.9 and $1.6 \mathrm{~mm} \mathrm{Hg}$, respectively) in patients treated with SGLT2 inhibitors, as compared to those receiving a placebo or other oral antidiabetic agents [24]. In the present study, although no significant improvements in blood pressure were observed in the entire cohort, the observations of significant decreases in SBP $(6.6 \mathrm{~mm} \mathrm{Hg})$ and DBP $(3.0 \mathrm{~mm}$ $\mathrm{Hg}$ ) in patients with poorly controlled blood pressure after 24 weeks of ipragliflozin treatment confirmed that this drug has an antihypertensive effect similar to those of other SGLT2 inhibitors. Thus, it is very interesting that we observed antihypertensive effects only in patients with poorly controlled blood pressure, i.e. there were no excessive antihypertensive effects with SGLT2 inhibitor administration in patients with well-controlled blood pressure. Furthermore, we observed a positive correlation between the changes in UACR and SBP, suggesting that ipragliflozin-mediated antihypertensive effects were associated with its beneficial effects in diabetic nephropathy.

In addition to their effects on sodium excretion, SGLT2 inhibitors might contribute to blood pressure control via the reduction of visceral fat or the regulation of various metabolic parameters. A previous study demonstrated that SGLT2 inhibitors reduced renal inflammation and facilitated microvascular system recovery in mice [25]; whereas another study showed that the treatment of patients with type 1 diabetes using SGLT2 inhibitors decreased pulse wave velocity [26]. Thus, antihypertensive effect of SGLT2 inhibitors appears to be attributed to, not only diuretic effect, but also complex effects such as nephron remodeling, suppression of arterial calcification and reduction of body weight [27].

\section{Conclusion}

The present study confirmed ipragliflozin to be beneficial for 
diabetic nephropathy and hypertension in patients with type 2 diabetes. The progression of diabetic nephropathy is associated with the onset of macrovascular disorders and consequently with the long-term outcomes of diabetic patients. Effective control of blood glucose, blood pressure, and serum lipid levels is critical for preventing and stopping the progression of diabetic nephropathy. Therefore, SGLT2 inhibitors might be a preventive treatment option for patients with type 2 diabetes complicated by diabetic nephropathy. Given the single arm and relatively small sample size of this study, future controlled trial, large-scale, long-term clinical studies, as well as basic experiments investigating the impact of these SGLT2 inhibitors on renal histopathology, are necessary.

\section{Acknowledgments}

We thank all investigators, trial staff, and participants.

\section{Conflicts of Interest}

The authors declare that they have no conflicts of interest concerning this article.

\section{Grant Support}

None.

\section{Financial Support}

No financial support for this study was provided by any company or foundation.

\section{References}

1. Bailey CJ, Gross JL, Pieters A, Bastien A, List JF. Effect of dapagliflozin in patients with type 2 diabetes who have inadequate glycaemic control with metformin: a randomised, double-blind, placebo-controlled trial. Lancet. 2010;375(9733):2223-2233.

2. Del Prato S, Nauck M, Duran-Garcia S, Maffei L, Rohwedder K, Theuerkauf A, Parikh S. Long-term glycaemic response and tolerability of dapagliflozin versus a sulphonylurea as add-on therapy to metformin in patients with type 2 diabetes: 4-year data. Diabetes Obes Metab. 2015;17(6):581-590.

3. Katsuyama H, Hamasaki H, Adachi H, Moriyama S, Kawaguchi A, Sako A, Mishima S, et al. Effects of Sodium-Glucose Cotransporter 2 Inhibitors on Metabolic Parameters in Patients With Type 2 Diabetes: A Chart-Based Analysis. J Clin Med Res. 2016;8(3):237-243.

4. Suzuki K, Mitsuma Y, Sato T, Anraku T, Hatta M. Comparison of Combined Tofogliflozin and Glargine, Tofogliflozin Added to Insulin, and Insulin Dose-Increase
Therapy in Uncontrolled Type 2 Diabetes. J Clin Med Res. 2016;8(11):805-814.

5. Yale JF, Bakris G, Cariou B, Yue D, David-Neto E, Xi L, Figueroa K, et al. Efficacy and safety of canagliflozin in subjects with type 2 diabetes and chronic kidney disease. Diabetes Obes Metab. 2013;15(5):463-473.

6. Cherney DZ, Perkins BA, Soleymanlou N, Maione M, Lai V, Lee A, Fagan NM, et al. Renal hemodynamic effect of sodium-glucose cotransporter 2 inhibition in patients with type 1 diabetes mellitus. Circulation. 2014;129(5):587597.

7. Wanner C, Inzucchi SE, Lachin JM, Fitchett D, von Eynatten M, Mattheus M, Johansen OE, et al. Empagliflozin and Progression of Kidney Disease in Type 2 Diabetes. N Engl J Med. 2016;375(4):323-334.

8. Osorio H, Coronel I, Arellano A, Pacheco U, Bautista R, Franco M, Escalante B. Sodium-glucose cotransporter inhibition prevents oxidative stress in the kidney of diabetic rats. Oxid Med Cell Longev. 2012;2012:542042.

9. Kojima N, Williams JM, Takahashi T, Miyata N, Roman RJ. Effects of a new SGLT2 inhibitor, luseogliflozin, on diabetic nephropathy in T2DN rats. J Pharmacol Exp Ther. 2013;345(3):464-472.

10. Nagata T, Fukuzawa T, Takeda M, Fukazawa M, Mori T, Nihei T, Honda K, et al. Tofogliflozin, a novel sodium-glucose co-transporter 2 inhibitor, improves renal and pancreatic function in $\mathrm{db} / \mathrm{db}$ mice. Br $\mathrm{J}$ Pharmacol. 2013;170(3):519-531.

11. Vallon V, Gerasimova M, Rose MA, Masuda T, Satriano J, Mayoux E, Koepsell H, et al. SGLT2 inhibitor empagliflozin reduces renal growth and albuminuria in proportion to hyperglycemia and prevents glomerular hyperfiltration in diabetic Akita mice. Am J Physiol Renal Physiol. 2014;306(2):F194-204.

12. Vallon V, Rose M, Gerasimova M, Satriano J, Platt KA, Koepsell H, Cunard R, et al. Knockout of Na-glucose transporter SGLT2 attenuates hyperglycemia and glomerular hyperfiltration but not kidney growth or injury in diabetes mellitus. Am J Physiol Renal Physiol. 2013;304(2):F156-167.

13. Komala MG, Panchapakesan U, Pollock C, Mather A. Sodium glucose cotransporter 2 and the diabetic kidney. Curr Opin Nephrol Hypertens. 2013;22(1):113-119.

14. Gilbert RE. Sodium-glucose linked transporter-2 inhibitors: potential for renoprotection beyond blood glucose lowering? Kidney Int. 2014;86(4):693-700.

15. Zinman B, Wanner C, Lachin JM, Fitchett D, Bluhmki E, Hantel S, Mattheus M, et al. Empagliflozin, Cardiovascular Outcomes, and Mortality in Type 2 Diabetes. N Engl J Med. 2015;373(22):2117-2128.

16. Kashiwagi A, Takahashi H, Ishikawa H, Yoshida S, Kazuta K, Utsuno A, Ueyama E. A randomized, double-blind, placebo-controlled study on long-term efficacy and safety of ipragliflozin treatment in patients with type 2 diabetes mellitus and renal impairment: results of the long-term ASP1941 safety evaluation in patients with type 2 diabetes with renal impairment (LANTERN) study. Diabetes Obes Metab. 2015;17(2):152-160.

17. Kashiwagi A, Kazuta K, Goto K, Yoshida S, Ueyama E, 
Utsuno A. Ipragliflozin in combination with metformin for the treatment of Japanese patients with type 2 diabetes: ILLUMINATE, a randomized, double-blind, placebocontrolled study. Diabetes Obes Metab. 2015;17(3):304308 .

18. Yamada K, Nakayama H, Yoshinobu S, Kawano S, Tsuruta $\mathrm{M}$, Nohara $\mathrm{M}$, Hasuo $\mathrm{R}$, et al. Effects of a sodium glucose co-transporter 2 selective inhibitor, ipragliflozin, on the diurnal profile of plasma glucose in patients with type 2 diabetes: A study using continuous glucose monitoring. J Diabetes Investig. 2015;6(6):699-707.

19. Iizuka T, Iemitsu K, Takihata M, Takai M, Nakajima S, Minami N, Umezawa S, et al. Efficacy and Safety of Ipragliflozin in Japanese Patients With Type 2 Diabetes: Interim Outcome of the ASSIGN-K Study. J Clin Med Res. 2016;8(2):116-125.

20. Iemitsu K, Iizuka T, Takihata M, Takai M, Nakajima S, Minami N, Umezawa S, et al. Factors Influencing Changes in Hemoglobin A1c and Body Weight During Treatment of Type 2 Diabetes With Ipragliflozin: Interim Analysis of the ASSIGN-K Study. J Clin Med Res. 2016;8(5):373-378.

21. Rahmoune H, Thompson PW, Ward JM, Smith CD, Hong $\mathrm{G}$, Brown J. Glucose transporters in human renal proximal tubular cells isolated from the urine of patients with noninsulin-dependent diabetes. Diabetes. 2005;54(12):34273434 .
22. Wakisaka M, Nagao T, Yoshinari M. Sodium Glucose Cotransporter 2 (SGLT2) Plays as a Physiological Glucose Sensor and Regulates Cellular Contractility in Rat Mesangial Cells. PLoS One. 2016;11(3):e0151585.

23. Bailey CJ, Iqbal N, T'Joen C, List JF. Dapagliflozin monotherapy in drug-naive patients with diabetes: a randomized-controlled trial of low-dose range. Diabetes Obes Metab. 2012;14(10):951-959.

24. Baker WL, Smyth LR, Riche DM, Bourret EM, Chamberlin KW, White WB. Effects of sodium-glucose co-transporter 2 inhibitors on blood pressure: a systematic review and meta-analysis. J Am Soc Hypertens. 2014;8(4):262275 e269.

25. Terami N, Ogawa D, Tachibana H, Hatanaka T, Wada J, Nakatsuka A, Eguchi J, et al. Long-term treatment with the sodium glucose cotransporter 2 inhibitor, dapagliflozin, ameliorates glucose homeostasis and diabetic nephropathy in db/db mice. PLoS One. 2014;9(6):e100777.

26. Cherney DZ, Perkins BA, Soleymanlou N, Har R, Fagan $\mathrm{N}$, Johansen OE, Woerle HJ, et al. The effect of empagliflozin on arterial stiffness and heart rate variability in subjects with uncomplicated type 1 diabetes mellitus. Cardiovasc Diabetol. 2014;13:28.

27. Maliha G, Townsend RR. SGLT2 inhibitors: their potential reduction in blood pressure. J Am Soc Hypertens. 2015;9(1):48-53. 